schenkt wird, ist wesentlicher Grund dafür, daß solches Strafrecht weithin für

legitim gehalten wird.

In Wirklichkeit dürfte das Strafrecht keine signifikanten Beiträge zur Bekämpfung von Terrorismus und Umweltkriminalität leisten. Zumindest hat es seine überwiegende Nützlichkeit empirisch bisher nicht belegt. Erst recht gilt dies für die Bekämpfung von AIDS. Alle diese Bereiche zeichnen sich vielmehr dadurch aus, daß bei ihnen - wie Hassemer jüngst bezüglich der Drogenkriminalpolitik ${ }^{24}$ und Herzog/Nestler-Tremel bezüglich der Aids-Kriminalpolitik ${ }^{25}$ gezeigt haben - ein hoher Problemlösungsbedarf dem - wirklichen oder vermeintlichen - Fehlen tauglicher Problemlösungsmöglichkeiten gegenübersteht. Diese Gemengelage aber ist, wie Hassemer betont, der Nährboden für eine symbolische Gesetzgebung, die sich darauf beschränkt, den Eindruck eines präsenten und entschlossen handelnden Gesetzgebers zu erwecken.

\title{
Michael Wunder Die Sterilisation Behinderter und der Schatten der Geschichte
}

»In Bonn waren wir uns nach eingehender Aussprache völlig darüber klar, daß es ein unsinniges Ding sei, heute Menschen als durch dieses Gesetz (Gesetz zur Verhütung erbkranken Nachwuchses von 1933, d.V.) zu Unrecht behandelte zu entschädigen und vielleicht morgen in die Notwendigkeit gesetzt zu sein, dieselben Menschen unter ein neues Eugenikgesetz zu stellen. "' Niemand geringeres als Friedrich von Bodelschwingh schrieb diesen Satz 1962 an den Bischof Dibelius von der Evangelischen Kirche Deutschland (EKD), nachdem er gerade aus Bonn zurückgekehrt war und sich dort gegen eine Wiedergutmachung für die Opfer der NSZwangssterilisation ausgesprochen hatte. Der Zusammenhang zwischen der Weigerung, die Zwangssterilisierten als Opfer des Nationalsozialismus anzuerkennen, und Bestrebungen, ein neuerliches Sterilisationsgesetz zu erlassen, drängt sich auch auf, wenn man die derzeitige Diskussion in Bonn betrachtet.

Nach jahrelangen Verzögerungen haben sich Mitte Januar 1988 die Koalitionsfraktionen und die SPD im Justizausschuß des Deutschen Bundestages darauf geeinigt, das "Gesetz zur Verhütung erbkranken Nachwuchses « nicht zum NS-Unrecht zu erklären. Eine solche Erklärung hätte den überlebenden Zwangssterilisierten - das Bundesfinanzministerium hatte 1985 ihre Zahl auf 88000 geschätzt - nicht nur die politische und moralische Anerkennung als Opfer des Faschismus gebracht, sondern auch Rentenansprüche. Beides wird jetzt den Betroffenen erneut verweigert. Der Justizausschuß beschloß als Vorlage für den Bundestag, daß lediglich die durchgeführten Zwangssterilisierungen Unrecht gewesen seien und als Maßnahme geächtet werden sollten. Die Zwangssterilisierten sollten erneut auf die seit 1980 bestehende Möglichkeit aufmerksam gemacht werden, 5000,- DM über den Härte-

24 Hassemer, JuS $1987,257 \mathrm{ff}$.

25 Herzog/Nestler-Tremel, StV $1987,361 \mathrm{f}$.

I Brief von Frıedrıch von Bodelschwingh vom 9. I I. 1962 an Bischof Dibelius von der EKD, Hauptarchıv der von Bodelschwinghschen Anstalten, Sig. 2/11-17. 
fond des Allgemeinen Kriegsfolgegesetzes zu beantragen. Zwangssterilisierte, die diese 5000,- DM einmal bezogen haben, sollen auch ausgeschlossen werden von Leistungen des neuen Härtefonds, den die Bundesregierung für die bisher »Vergessenen NS-Opfer « einrichten will. In den Anhörungen zur gesetzlichen Neuregelung der sogenannten Wiedergutmachung haben sich 1986 und 1987 in Berlin, Hamburg und Bonn erstmals Zwangssterilisierte öffentlich zu Wort gemeldet. Sie haben darüber berichtet, wie stark die Sterilisation ihr Selbstwertgefühl herabminderte und sie zu Menschen zweiter Klasse machte, worüber sie über Jahrzehnte nicht sprechen konnten. Die durch die Bonner Beschlüsse bestärkte Abweisungsbürokratie der Wiedergutmachungsämter wird die Betroffenen erneut entmutigen und die öffentliche Auseinandersetzung über das Schicksal der Zwangssterilisierten erschweren.

Zeitgleich mit der Debatte über die Neuregelung der Wiedergutmachung wurde im Bundesjustizministerium ein denkwürdiger Diskussionsentwurf zur Reform des Vormundschafts- und Pflegschaftsrechtes erarbeitet. ${ }^{2}$ Statt der bisherigen Totalentmündigungen sollen in Zukunft nach dem Erforderlichkeitsgrundsatz nur noch sogenannte Betreuungsschaften in gerichtlich festgelegten Aufgabenbereichen angeordnet werden. Die Rechte der "Unter-Betreuung-Gestellten « sollten gestärkt werden. Gleichzeitig befindet sich in diesem in seiner Intention sicherlich begrüßenswerten Reformgesetz auch die Legalisierung der Sterilisation sogenannter nicht einwilligungsfähiger Personen. Die Entwurfsautoren halten es zwar für "problematisch ..., die Vormundschaftsreform mit einer Sterilisationsdebatte zu verbinden ${ }^{3}$ und damit ggf. ihr gesamtes Reformprojekt zu gefährden, fühlen sich jedoch dem jahrzehntelangen Drängen verschiedener Verbände und Berufsgruppen nach einem Sterilisationsgesetz verpflichtet. Nachdem die Lebenshilfe diese Forderung über Jahre hin propagiert hat, in letzter Zeit aber deutlich moderater geworden ist ${ }^{4}$, fordern dies jetzt vor allem die Bundesärztekammer's und die Medizinrechtler, die sich im "Einbecker-Expertengespräch « zusammengeschlossen haben. ${ }^{6}$

Es wäre also einseitig, erklärte man die erneute Weigerung, die Zwangssterilisierten als Opfer des Faschismus anzuerkennen, mit dem finanzpolitischen Argument Stoltenbergs, dies würde I Milliarde DM kosten. Von mindestens ebenso großem Gewicht war sicherlich die Einsicht, daß man nicht gleichzeitig das Erbgesundheitsgesetz zum Unrechtsgesetz erklären kann, während man ein neues Sterilisationsgesetz vorbereitet. Die vorgeschlagene Gesetzesänderung des BGB soll folgenden Wortlaut haben:

\$ $\$ 1905$

(I) In eine Sterilisation des Betreuten, in die dieser nicht einwilligen kann, kann der Betreuer nur einwilligen, wenn

I. die Sterilisation dem Willen des Betreuten nicht widerspricht,

2. der Betreute auf Dauer einwilligungsunfähig bleiben wird,

3. anzunehmen ist, daß es ohne die Sterilisation zu einer Schwangerschaft kommen würde,

4. anzunehmen ist, daß ein Abbruch dieser Schwangerschaft gem. $\$ 2$ I 8a Absatz 1, Absatz 2 Nummer 1,3 des Strafgesetzbuches nicht strafbar wäre und

ร. die Schwangerschaft nicht durch andere zumutbare Mitte] verhindert werden kann.

2 Diskussionsteilentwurf: Gesetz über die Betreuung Volljähriger (Betreuungsgesetz - BtG -), herausgegeben vom Bundesminister der Justiz, November 1987 , Bundesanzeiger.

3 A. a. O. Seite 68

4 Vgl. Neuer-Miebach, Therese, u.a. (Hrsg.), Schwangerschaftsverhütung bei Menschen mit geistiger Behınderung - notwendig, möglich, erlaubt, Große Schriftenreihe der Lebenshilfe, Band 18, Marburg/ Lahn 1987, Sette $231 \mathrm{ff}$.

5 Bundesärztekammer, Zulässıgkeıt eıner Sterilisatıon geıstıg Behınderter aus eugenıscher oder sozıaler Indikation, Hamburger Ärzteblatt r/87.

6 Zweites Einbecker-Expertengespräch der Gesellschaft für Medizinrecht, Empfehlungen zur Sterilisatıon gerstig Behinderter vom 19./21.6. 1987. 
(2) Die Einwilligung bedarf der Genehmigung des Vormundschaftsgerichts. Die Sterilisation darf erst 2 Wochen nach Wirksamkeit der Genehmigung durchgeführt werden. «7

Eine Sterilisation Minderjähriger soll durch eine entsprechende Änderung des BGB $\left(\$ 16_{3} \mathrm{I}\right)$ verhindert werden. Die Autoren distanzieren sich in ihren Erläuterungen zu dem vorgeschlagenen Gesetzestext deutlich von verschiedenen Motiven für eine Sterilisationsgesetzgebung. So lehnen sie z. B. Sterilisation im "Interesse der Allgemeinheit«, die die Geburt von Behinderten als Belastung empfinden könnte, ab. Ebenso lehnen sie eine Sterilisation im "Interesse von Verwandten « ab oder im "Interesse des ungezeugten Kindes«. Ein solcher Gedanke, daß es für manche Menschen besser sei, nicht geboren worden zu sein, dürfte niemals anerkannt werden. Soweit kann man den klaren und wohltuenden Erläuterungen durchaus folgen. Im weiteren Text werden dann aber die Haken und Ösen des Entwurfs mit schönfärberischen Worten zugedeckt.

\section{Problem I: Wer ıst »einwilligungsunfäbig«?}

Hierzu schweigt sich der Entwurf aus. Einwilligungsfähigkeit könnte bedeuten, den Vorgang "Sterilisation" nicht als einen Eingriff zu begreifen, der endgültig zur Kinderlosigkeit führt. In der Rechtsprechung wird die Einwilligungsfähigkeit allerdings häufig als »konkrete Einsichts- und Urteilsfähigkeit ${ }^{8}{ }^{2}$ definiert. Mit anderen Worten: Was Einwilligungsfähigkeit dann bezüglich der Urteilsfähigkeit alles bedeuten kann, eröffnet ein weites Feld von Interpretationsmöglichkeiten und je nach gesellschaftlichem Umfeld Bewertungen durch den jeweiligen Gutachter. Fest steht auf jeden Fall, das Nadelöhr der Sterilisationsgesetzgebung wird das ärztliche Gutachten über die Einwilligungsunfähigkeit sein. Ein Gutachten im übrigen, zu dem der Betroffene und die Betroffene auch zwangsweise vorgeführt werden können, wie die Autoren an anderer Stelle ihres Diskussionsentwurfs ausführen. ${ }^{9}$

\section{Problem 2: Was ist eine "Zwangssterilisation«?}

Die Entwurfsautoren argumentieren, daß eine Sterilisation gegen den erkennbaren, geäußerten Willen der betroffenen Person eine Zwangssterilisation ist, die in jedem Fall unterlassen werden muß. Eine Sterilisation obne den Willen der betroffenen Person sei allerdings kein Zwangseingriff und deshalb nach der vorgeschlagenen Gesetzesänderung auch legal. Was aber juristisch so glatt formulierbar ist, muß noch lange nicht mit der Praxis übereinstimmen, insbesondere mit der Lebenspraxis geistig behinderter Menschen.

Viele Menschen mit geistiger Behinderung erscheinen entscheidungslabil, weil es für sie schwierig ist, sich die Folgen von etwas vorzustellen, was erst in der Zukunft geschehen wird und was zum gegenwärtigen Zeitpunkt nur mit Worten zu beschreiben ist. Hinzukommt, daß es für viele von ihnen eine besondere Schwierigkeit darstellt, auf Aufforderung hin, ihren »natürlichen Willen«zu äußern. Auf den Gutachter oder den Richter könnten sie also durchaus den Eindruck der Willenlosigkeit machen, aber trotzdem eine gefühlsmäßige Abwehr gegen eine angekündigte Handlung oder, was noch wichtiger ist, während einer solchen Handlung haben. Sie könnten also durchaus nach einer für die Richter und Gutachter ergebnislosen Befragung die Einlieferung ins Krankenhaus später als angstvoll erleben, als persönlich degradierend oder sie in ihren Rechten beschneidend.

\footnotetext{
7 Diskussionsteilentwurf, Sette 4.

8 Zit. nach Neuer-Miebach, Sette 238.

9 Diskussionsteilentwurf, Seıte 9.
} 
Im Gesetzesentwurf wird also Schönfärberei mit Worten betrieben und eine Trennlinie hochgezogen, die in Wirklichkeit überhaupt keine ist. Das Kriterium »geäußerter, natürlicher Wille« ist viel zu hoch und verkennt, daß eine $Z$ wangssterilisation auch dann vorliegt, wenn der Eingriff im Zustand einer juristisch gesehenen Willenlosigkeit geschieht. Übrigens: Auch eine herbeigeredete Zustimmung $\mathrm{zu}$ einer Sterilisation ist keine Garantie dafür, daß es sich hierbei nicht um eine Zwangsmaßnahme handelt. Ich habe in meiner beruflichen Tätigkeit eine Reihe von geistig behinderten Frauen kennengelernt, die vor der Sterilisation befragt wurden und dem Eingriff sogar zustimmten, die damit verbundene Kinderlosigkeit aber als zwangsweise erzeugt erlebt haben und als größtes Problem ihres Lebens.

\section{Problem 3: Sterilisation als Prophylaxe vor Schwangerschaftsabbrüchen.}

Der Kernpunkt der vorgeschlagenen Neuregelung ist die Argumentation, daß durch eine Sterilisation eine Notlage verhindert werden soll, die sonst zu einem straffreien Schwangerschaftsabbruch nach $\ 2$ I 8 a StGB führen könnte. $\ 218 a$ beschreibt Notlagen von Schwangeren, in denen das Austragen des Kindes als unzumutbar anerkannt wird und deshalb der Schwangerschaftsabbruch straffrei ist. Die strenge medizinische Indikation besteht dann, wenn die Schwangerschaft zu einer konkreten Gefahr für das Leben der Schwangeren führen würde ( $\$ 218 a \mathrm{Absatz}$ I). Die erweiterte medizinische Indikation besteht dann, wenn die konkrete und ernstliche Gefahr einer schwerwiegenden Beeinträchtigung des körperlichen und seelischen Gesundheitszustandes für die Schwangere besteht. Diese Gefahr kann laut $\$ 2$ I8a dann vorliegen, wenn das Kind behindert ist $(\$ 2$ I 8 a Absatz 2 Nr. I) oder wenn die Schwangerschaft, die Geburt und die Betreuung des Kindes eine soziale Notlage für die Frau entstehen ließen ( $\$ 2$ 2 8 a Absatz 2 Nr. 3 ).

Wird diese Argumentationslogik des $\$ 2$ I 8 a StGB auf die Sterilisation übertragen, so entstehen unvermeidbar zwei systematische Fehler:

- Der Schwangerschaftsabbruch nach $\$ 218 a$ ist immer nur dann möglich, wenn es tatsächlich zu einer Schwangerschaft gekommen ist. Die Übertragung der Indikationen auf einen prophylaktischen Eingriff, der alle zukünftigen Schwangerschaften vermeiden soll, suggeriert, daß die Anerkennung einer Notlage für das gesamte Leben einer Frau möglich sei. Ist dies in bestimmten Fällen der strengen medizinischen Indikation wahrscheinlich möglich, müssen hier jedoch deutliche Zweifel bezüglich der sozialen Indikation angemeldet werden. Wenn heute Gutachter behaupten, eine geistig behinderte Frau sei nicht fähig, ihr Kind auch $\mathrm{zu}$ erziehen, so kann dies z. B. nach einer bestimmten Förderungsphase in ein paar Jahren absolut unzutreffend sein.

- Die Übertragung der Indikationen des $\ 218 a$ auf die Sterilisation läßt vergessen, daß das Anerkennungsverfahren nach $\$ 218 \mathrm{a}$ in der Regel von der betroffenen schwangeren Frau selbst in Gang gesetzt wird und sie entscheidet, ob es bei Anerkennung einer Notlage auch tatsächlich zum Abbruch kommt. Bei einem Sterilisationseingriff, wo die Indikationen des $\$ 2 \mathrm{I} 8 \mathrm{a}$ als Begründung herangezogen werden, stellen jedoch Dritte die sogenannte Notlage oder die Unzumutbarkeit fest. Vergleichbar ist diese ersatzweise Feststellung nur mit der auch bisher praktizierten ersatzweisen Einwilligung zum Schwangerschaftsabbruch bei sog. nicht einwilligungsfähigen, entmündigten Frauen durch ihren Vormund. Hier wie bei der geplanten Ausweitung auf die Sterilisation bedeutet dies zum Beispiel für die »eugenische Indikation « $(\$ 2$ I 8a Abs. 2 Nr. I) eben nicht, daß die Betroffene selbst die Unzumutbarkeit, ein behindertes Kind zu bekommen, ins Feld führt, sondern daß Gutachter, Ärzte und Richter dies tun. Was liegt da näher, als daß 
diese nach ihren eigenen Maßstäben urteilen statt nach denen der Frau? Was sich heute dabei bereits im Bereich der ersatzweisen Einwilligung zum Schwangerschaftsabbruch abspielt, wird bei einer Ausweitung auf die Sterilisation in großem Maßstab stattfinden: Aus der Feststellung der Zumutbarkeit für die einzelne Frau wird die Feststellung der Zumutbarkeit für die Gesellschaft.

Noch deutlicher wird der Wechsel des Entscheidungsmotivs im Falle der sozialen Indikation. Hier sprechen selbst die Entwurfsautoren schon davon, daß die Notlage dann bestehe, wenn »eine behinderte Frau aufgrund der Schwere ihrer Behinderung nie in der Lage sein wird, ihr auch nicht behindertes Kind aufzuziehen. ${ }^{10}$ Welche geistig behinderte Frau kann ihr Kind schon ohne weitere Hilfe erziehen? Oder: Welcher Gutachter ist heute bereit, einer geistig behinderten Frau das Gegenteil zu bescheinigen? Die »Unzumutbarkeit für die Gesellschaft«, die Kinder geistig behinderter Eltern erziehen zu müssen, lugt hier aus jeder Zeile. Man kann es auch anders ausdrücken: Was der $\$ 2$ r 8 a StGB bei aller Doppelbödigkeit und Halbherzigkeit an Stärkung der Autonomie der betroffenen Frauen gebracht hat, wird bei der Übertragung auf die Sterilisation wieder in das Gegenteil verkehrt. Aus "individueller Zumutbarkeit « wird eine "gesellschaftliche Zumutbarkeit«. Was vorher individuelle Erleichterung und Vergrößerung der Autonomie war, kann jetzt Planungsgrundlage für eine bestimmte Sozial- und Bevölkerungspolitik werden.

Und noch etwas fällt auf: Die Übertragung der Indikationen des $₫$ 218a StGB auf die Sterilisation hat zur Folge, daß von einem solchen Gesetz fast ausschließlich Frauen betroffen sind. Hinsichtlich der Männer kommen die Entwurfsautoren in einen unübersehbaren Argumentationsnotstand. Wie soll nämlich die zukünftige Notlage einer Schwangeren eingeschätzt werden, wenn es um die Sterilisation eines Mannes geht? Die einzige logische Möglichkeit besteht, wenn ein geistig behinderter Mann ein festes Verhältnis mit einer geistig behinderten Frau hat, für die dann die Gutachter und Richter die angesprochenen Indikationen feststellen. Ein geistig behinderter Mann mit häufig wechselnden oder nicht bekannten Partnerinnen kann demnach nicht sterilisiert werden. Den Gutachtern und Richtern wird auch die Feststellung der potentiellen Partnerinnen geistig behinderter Männer viel zu aufwendig und schwierig sein, so daß dieses Gesetz fast ausschließlich die geistig behinderten Frauen treffen wird.

\section{Legitimation durch Verfabren}

Der Entwurf hat bestechende Verfahrensvorschläge, die alle Betreuungsangelegenheiten betreffen sollen:

- Geschäftsunfähige werden durchaus als verfahrensfähig anerkannt. Sie können also Widerspruch einlegen oder sich anderweitig am Verfahren direkt beteiligen. $\mathrm{Zu}$ ihrer Unterstützung werden ihnen Verfahrenspfleger beigeordnet, die sie beraten.

- Wichtige Entscheidungen des Betreuers, z. B. auch seine Einwilligung in die Sterilisation, bedürfen der Genehmigung durch das Vormundschaftsgericht. Selbstherrlichkeiten einzelner Betreuer sollen also ausgeschlossen werden.

Bei der Legalisierung der Sterilisation ohne persönliche Einwilligung können diese Verfahrensvorschläge jedoch nicht beruhigen. Die Idee, Sterilisationseingriffe durch Verfahren zu legitimieren, ist alt. Sie konnte damals und sie wird heute die 
Menschen nicht vor zwangsweisen Eingriffen in ihre persönliche Integrität bewahren.

\section{Ausblick}

Die Sterilisationsdebatte war auch historisch gesehen immer das Sprungbrett für weitere Eingriffe in das Lebensrecht bestimmter Gruppen in der Gesellschaft. In der Sterilisationspraxis der dreißiger Jahre wurde die Ausweitung auf immer mehr Gruppen und auf den zwangsweisen Schwangerschaftsabbruch betrieben. Dies waren keine Mißbräuche des bestehenden Gesetzes, sondern Ausweitungen, die sich aus der inneren Logik des Gesetzes ergaben. Es wird deshalb wenig Sinn haben, eine mißbrauchssichere Formulierung für die heutige Gesetzgebung zu fordern. Die Ausweitung der Kategorie »einwilligungsunfähig « ist schon jetzt im Entwurf unvermeidbar angelegt, weil Gutachter in ihrer ärztlichen Denkweise hierüber entscheiden. Der Wechsel der Blickrichtung von der "Zumutbarkeit für die Schwangere zu einer "Zumutbarkeit für die Gesellschaft « ist ebenfalls jetzt schon in der inneren Logik der Argumentation des Entwurfes angelegt, da nicht die Betroffenen, sondern Dritte hierüber befinden. Es darf nicht verkannt werden, daß zeitgleich mit dem Versuch, die Sterilisation ohne Einwilligung zu legalisieren, eine verstärkte öffentliche Debatte über die Legalisierung der aktiven Sterbehilfe eingesetzt hat. Es gibt nicht nur eine philosophische und gedankliche Verbindung beider Debatten: Die eine Debatte betrifft die Verfügbarkeit des Lebens an seinem Anfang, die andere Debatte die Verfügbarkeit des Lebens an seinem Ende. Die Sterbehilfedebatte beschäftigt sich darüberhinaus mit vielen ähnlichen Themen wie die Sterilisationsdebatte. Auch hier steht die Erkennbarkeit eines Willens, die Mutmaßung über diesen Willen und die Indikation, was ein qualitätsvolles oder zumutbares Leben ist, im Mittelpunkt. Mit anderen Worten: Sollte die Sterilisation ohne Einwilligung legalisiert werden, käme dies einer Weichenstellung für die spätere Legalisierung der aktiven Sterbehilfe gleich. Es lohnt sich also heute ein breites gesellschaftliches Bündnis gegen diesen Gesetzentwurf zu versuchen, zu dem Behinderte und ihre Verbände, Frauengruppen, Zwangssterilisierte aus der NS-Zeit, die Kirchen usw. angesprochen werden sollten. Ziel muß dabei sein, daß das Justizministerium den Sterilisationsteil aus dem Gesetzentwurf streicht.

Eine gefährliche Nebenwirkung der jetzigen Gesetzesdiskussion ist, daß sie den Blick verstellt und die Aktivitäten für neue Lebensformen für geistig behinderte Menschen mit ihren Kindern lähmt. Es gibt einige wenige Wohngruppen und Wohnformen, in denen geistig behinderte Menschen auch schon heute mit ihren Kindern zusammen leben können. Eine Entscheidung gegen ein neues Sterilisationsgesetz kann nur durchgesetzt werden, wenn es gelingt, mehr solche Wohnformen zu schaffen und sie öffentlich zu propagieren. Daß geistig behinderte Menschen Kinderwünsche haben und fähig werden sollten, Kinder auch zu bekommen und mit Hilfe zu erzichen, sollte zur Normalität werden. 